\title{
Inhibitory effect of endothelin A receptor blockade on tumor growth and liver metastasis of a human gastric cancer cell line
}

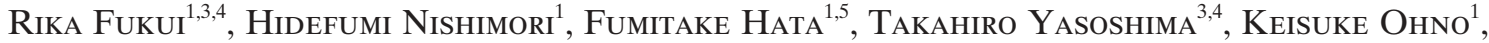 \\ Yoshiyuki Yanai $^{2,3,4}$, Kenjiro Kamiguchi ${ }^{2}$, Ryuichi Denno ${ }^{1}$, Noriyuki Sato $^{2}$, and Koichi Hirata ${ }^{1}$ \\ ${ }^{1}$ First Department of Surgery, Sapporo Medical University School of Medicine, South-1, West-16, Chuo-ku, Sapporo 060-8543, Japan \\ ${ }^{2}$ First Department of Pathology, Sapporo Medical University School of Medicine, Sapporo, Japan \\ ${ }^{3}$ Institute of Gastroenterology, Shinsapporo Keiaikai Hospital, Sapporo, Japan \\ ${ }^{4}$ Department of Surgery, Shinsapporo Keiaikai Hospital, Sapporo, Japan \\ ${ }^{5}$ Department of Surgery, Eniwa Daiichi Hospital, Eniwa, Japan
}

\begin{abstract}
Background. With metastatic progression, gastric cancer is incurable. Using a DNA microarray, we performed differential gene expression analysis of established highly metastatic gastric cancer cell lines and compared the findings with those from a low-metastatic parental cell line. The results demonstrated that the endothelin A receptor (ET-A) gene was the only one from the highly metastatic cell lines that was generally up-regulated.

Methods. To investigate the role that ET-A plays in gastric cancer metastasis, we studied the effect of an ET-A-selective antagonist, YM598, on cell proliferation, tumor growth, and liver metastasis of the highly liver metastatic cell line AZ-H5c, established from the low metastatic human gastric cancer cell line AZ-521.

Results. An in vivo study using nude mice demonstrated that YM598 had a significant growth inhibition effect on AZ-H5c at doses of $0.5-10.0 \mathrm{mg} / \mathrm{kg}$. The liver metastatic rate was also significantly reduced by YM598: control, $83.3 \% ; 1 \mathrm{mg} / \mathrm{kg}$ dosage, $16.7 \% ; 10 \mathrm{mg} / \mathrm{kg}, 20 \%$; and pretreatment at $1 \mathrm{mg} / \mathrm{kg}$, $16.7 \%$. There was no evidence of gross toxicity resulting from the YM598 treatment.

Conclusion. The ET-A blockade by YM598 had a strong inhibitory effect against tumor growth and liver metastasis of the gastric cancer cell lines. These data suggest that YM598 has potential as a novel therapeutic agent for inhibiting liver metastasis of gastric cancer.
\end{abstract}

Key words Endothelin A receptor - Gastric cancer · Liver metastasis $\cdot$ Metastasis-related gene $\cdot$ Microarray analysis

\section{Introduction}

Gastric cancer is the main cause of cancer death in Japan. In particular, more efficacious treatment modalities are needed for metastatic gastric cancer. In this

Offprint requests to: R. Fukui

Received: November 15, 2006 / Accepted: April 15, 2007 study, to determine the metastasis-related genes of gastric cancer, we analyzed the differential gene expression of established human gastric cancer cell lines - AZH5c, a highly liver metastatic cell line; AZ-P7a, a cell line with high peritoneal dissemination; AZ-L5G, a highly lymph node metastatic cell line - and compared the findings with those from AZ-521, a parental low metastatic cell line. The results revealed that the ET-A gene was the only one of three highly metastatic cell lines that was generally up-regulated, in contrast to the AZ-521 line. Thus, we evaluated the possibility that ET-A might play an important role in the mechanism of gastric cancer metastasis and that the ET-A blockade could have an antimetastatic effect. We conducted in vitro and in vivo experiments using a non-peptideselective ET-A antagonist, YM598 (Astellas Pharma, Tokyo, Japan). The present study demonstrates the inhibitory effect of ET-A blockade on the cell proliferation, tumor growth, and liver metastasis of gastric cancer.

\section{Materials and methods}

\section{Animals}

Athymic female BALB/c nu/nu mice used; they were 5-7 weeks old and weighed 20-22 g. They originated from the Central Institute for Experimental Animals (Kawasaki, Japan) and were purchased from Clea Japan (Tokyo, Japan). The mice were maintained in a laminar airflow cabinet under specific pathogen-free conditions and were provided with sterile food and water. All experiments were performed according to institutional ethical guidelines on animal care.

\section{Cell lines and cell culture}

A human gastric cancer cell line, AZ-521 (obtained from the Japanese Cancer Research Resources Bank, 
Tokyo, Japan), and established highly metastatic variants, AZ-H5c (a liver metastatic line), AZ-P7a (a peritoneal dissemination line), and AZ-L5G (a lymph node metastatic line), were used in this study [1-3]. These three highly metastatic variants were established in our institution as previously described [1-3]; their metastatic rates are presented in Fig. 1. The cell lines were maintained in a humidified atmosphere of $95 \%$ air and $5 \% \mathrm{CO}_{2}$ at $37^{\circ} \mathrm{C}$ by culturing in an RPMI 1640 medium (Asahi Techno Glass, Funabashi, Japan) supplemented with $10 \%$ fetal bovine serum (FBS) (GIBCO, Grand Island, NY, USA), tylosin tartrate $8 \mu \mathrm{g} / \mathrm{ml}$ (SigmaAldrich, St. Louis, MO, USA), penicillin 50 units $/ \mathrm{ml}$, and streptomycin $50 \mathrm{mg} / \mathrm{ml}$ (GIBCO). The cells were passaged and expanded by trypsinization of cell monolayers followed by replating every $4-5$ days. The culture medium was changed every 2-3 days.

\section{Target preparation}

Differential gene expression among the four cell lines was measured. First, poly $(\mathrm{A})^{+} \mathrm{mRNA}$ was extracted from cultured cell pellets using a Fast Track mRNA Isolation Kit (Invitrogen, Carlsbad, CA, USA) according to the manufacturer's instructions and quantified spectrophotometrically. Double-stranded cDNA was synthesized from purified poly $(\mathrm{A})^{+} \mathrm{mRNA}$ using the T7- $(\mathrm{dt})_{24}$ primer (5'-GGCCAGTGAATTGTAAGTA ATACGACTCACTATAGGGAGGCGG-(dt) $\left.{ }_{24}-3^{\prime}\right)$ and a Superscript Kit (Invitrogen). Double-stranded

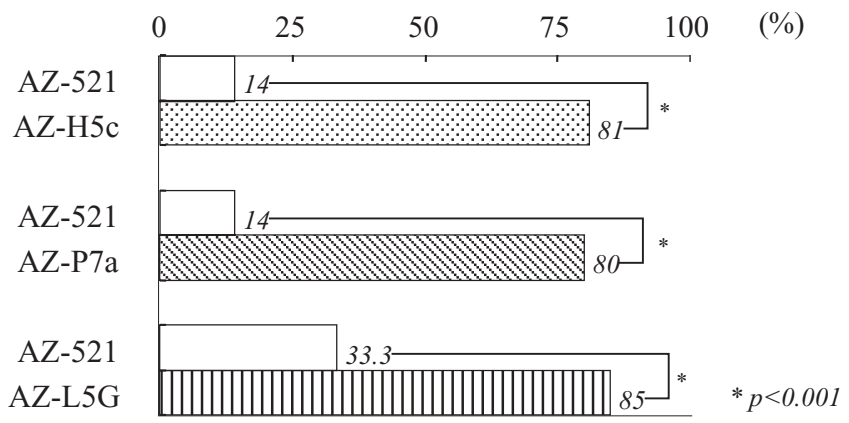

Fig. 1. To evaluate the metastatic potential, cultured cells from each cell line were inoculated into the spleen (AZ-H5c, $5 \times 10^{6}$ cells), peritoneal cavity (AZ-p7a, $1 \times 10^{7}$ cells), and perigastric wall (AZ-L5G, $5 \times 10^{6}$ cells) of nude mice. Three weeks after inoculation, the mice were sacrificed and examined for metastasis. The three established cell lines demonstrated significantly higher metastatic ability than the parental AZ-521 cells. The liver metastatic rate of AZ-H5c was $81 \%$; the peritoneal dissemination rate of AZ-P7a was $80 \%$; and the lymph node metastatic rate of AZ-L5G was $85 \%$. In contrast, the metastatic rates of AZ-521 were $14 \%$ for the liver, $14 \%$ for the peritoneal cavity, and $33.3 \%$ for the lymph nodes. $* P<0.001$ versus control
cDNA was purified by phenol/chloroform extraction with phase lock gels. Biotin-labeled antisense cRNA (target) in an in vitro transcription reaction (IVT) was produced using the ENZO BioArray RNA Transcript Labeling Kit (Affymetrix, Santa Clara, CA, USA). The IVT product (cRNA) was then cleaned up using QIAGEN RNeasy Columns (Qiagen, Hilden, Germany) according to the manufacturer's instructions. Subsequently, the cRNA was fragmented for target preparation.

Target hybridization and washing, staining, and scanning probe arrays

A hybridization cocktail was prepared using a Gene Chip eukaryotic Hybridization Control Kit (Affymetrix). The cocktail was then hybridized on the Gene Chip Human Cancer G110 Array (Affymetrix) during a 16 -h incubation in a $45^{\circ} \mathrm{C}$ oven. Before hybridization to the G110 array, the target RNA quality was determined by hybridization to the Affymetrix Test 2 Array.

Immediately following hybridization, the hybridized probe array was subjected to an automated washing and staining protocol on the fluid station according to the Gene Chip Fluidics Station 400 User's Manual. Each probe array was scanned twice. The computer workstation automatically overlaid the twice-scanned images and averaged the intensities of each probe cell for the greatest assay sensitivity.

\section{Array data analysis}

Data were analyzed using Microarray Suite 4.0 software (Affymetrix). The software calculated the average intensity of the signals from each probe array and then applied the selected array algorithm to determine the expression levels for each gene. The average expression level of each gene in each cell line was calculated, and the ratio of the average expression level between the two cell lines was then calculated. Comparative analysis of the parental cell line, metastatic cell lines, and each metastatic cell line was performed. The cutoff value was set at 3.0 for the ratio.

\section{Detection of $m R N A$ s by RT-PCR}

Total RNA was extracted from the four cell lines with the QIAGEN RNeasy Mini Kit according to the manufacturer's instructions. All RNA samples were quantified by spectrophotometry and stored at $-80^{\circ} \mathrm{C}$ until processed for reverse transcription (RT). First-strand cDNA was synthesized from $1 \mu \mathrm{g}$ of the total RNA using the Super Script Choice System for cDNA Synthesis in $19 \mu \mathrm{l}$ of reaction volume, according to the instructions 
provided. The synthesized first-strand cDNA was amplified by the polymerase chain reaction (PCR) in a final reaction volume of $50 \mu \mathrm{l}$, containing $1 \mu \mathrm{l}$ of cDNA, $0.5 \mu \mathrm{M}$ of each oligonucleotide primer, $0.2 \mathrm{mM}$ of each dNTP, 2.5 units of Taq DNA polymerase, and $5 \mu$ l of a $10 \times$ PCR buffer. The sequences of primers used were (sense) 5'-TTT GCC TCA AGA TGG AAA CC-3' and (antisense) 5'-TGT GGG CAA TAG TTG TGC AT-3' for ET-A (223bp); and (sense) 5'-GAG TCA ACG GAT TTG GTC GT-3' and (antisense) 5'-TTG ATT TTG GAG GGA TCT CG-3' for GAPDH (238bp). Amplification was carried out in a Gene Amp PCR system 9700 (Applied Biosystems, Foster City, CA, USA), and the PCR conditions were as follows: $94^{\circ} \mathrm{C}$, 5 min for 1 cycle; $94^{\circ} \mathrm{C}$ for $30 \mathrm{~s}$ (denaturation), $55^{\circ} \mathrm{C}$ for $30 \mathrm{~s}$ (annealing), and $72^{\circ} \mathrm{C}$ for $30 \mathrm{~s}$ (extension) for 25 cycles followed by $7 \mathrm{~min}$ final extension at $72^{\circ} \mathrm{C}$. The amplification products were electrophoresed on $1.0 \%$ $(\mathrm{w} / \mathrm{v})$ agarose gels and visualized by ethidium bromide staining under ultraviolet light.

\section{Cell proliferation assay in vitro}

AZ-521 and AZ-H5c cultured in an RPMI 1640 medium were harvested at $80 \%$ confluence and seeded at a plating density of $5 \times 10^{5}$ cells/well in six-well plates. They were then cultured for $24 \mathrm{~h}$, and the culture medium was changed to an FBS-free medium supplemented with YM598. We studied seven concentrations of YM598 from 0 to $0.2 \mathrm{mM}$. Following culture with YM598 for $48 \mathrm{~h}$, the cells were dispersed by trypsinization and counted in a hemocytometer. The experiments were performed in triplicate and repeated twice.

\section{Assay of tumor growth in vivo}

Five-week-old female (nu/nu) mice were given injections subcutaneously (s.c.) in the back with $1 \times 10^{7} \mathrm{AZ}$ H5c cells suspended in $100 \mu \mathrm{l}$ of PBS. The mice were then randomly divided into four groups of eight animals each and received the following treatments: group 1 was injected intraperitoneally (i.p.) with $100 \mu \mathrm{l}$ of PBS every day for 3 weeks after inoculation; group 2 was injected in the same way with a solution containing $0.01 \mathrm{mg}$ of YM598 dissolved in PBS at a dose of $0.5 \mathrm{mg} / \mathrm{kg}$; group 3 was injected in the same way with a solution containing $0.02 \mathrm{mg}$ of YM598 dissolved in PBS at a dose of $1 \mathrm{mg} / \mathrm{kg}$; and group 4 was injected in the same way with a solution containing $0.2 \mathrm{mg}$ of YM598 dissolved in PBS at a dose of $10 \mathrm{mg} / \mathrm{kg}$. These doses were chosen according to the supplier's recommendations [4]. Treatment started on the same date of the inoculation. Tumor volume was measured with a caliper and calculated as (length $\times$ width $\times$ height) $/ 2$. Mouse body weight was measured once a week.
Assay of liver metastasis in vivo

Five-week-old female (nu/nu) mice were given injections of $5 \times 10^{6}$ AZ-H5c cells suspended in $100 \mu$ l of PBS in the spleen. The mice were then randomly divided into four groups of five or six animals each and received the following treatments: group 1 was injected with $100 \mu \mathrm{l}$ of PBS i.p. every 2 days for 3 weeks after inoculation; group 2 was injected with YM598 every 2 days for 3 weeks after inoculation at a dose of $1 \mathrm{mg} / \mathrm{kg}$ i.p.; group 3 was injected with YM598 in the same way at a dose of $10 \mathrm{mg} / \mathrm{kg}$; and group 4 was injected with YM598 at a dose of $1 \mathrm{mg} / \mathrm{kg}$ starting 2 days before inoculation. The mice were sacrificed 3 weeks later, and the liver metastasis rate was calculated.

\section{Statistical analysis}

The statistical difference was determined by Student's $t$-test. $P<0.05$ was considered significant.

\section{Results}

\section{Microarray assay}

A differential gene expression analysis using a DNA microarray demonstrated that many genes were up- and down-regulated in highly metastatic cell lines, in contrast to the parental cell line AZ-521. In these differentially expressed genes, the ET-A gene was the only one of three highly metastatic variants that was generally up-regulated, in contrast to the AZ-521 line. The ratios of the ET-A mRNA expression level in comparison with AZ-521 were 30.1 (AZ-H5c), 57.3 (AZ-P7a), and 47.8 (AZ-L5G) (Table 1).

\section{Detection of $m R N A$ s by $R T-P C R$}

RT-PCR revealed that the mRNA expression of ET-A in AZ-H5c, AZ-p7a, and AZ-L5G was higher than that of the low metastatic AZ-521. The ET-A mRNA upregulation in AZ-H5c, AZ-p7a, and AZ-L5G was confirmed (Fig. 2).

\section{Effect of YM598 on AZ-521 and AZ-H5c cell proliferation in vitro}

After a 48-h incubation with YM598 in the $0.01-0.05 \mathrm{mM}$ concentration, the number of viable cells of AZ-521 and AZ-H5c decreased. In the $0.1-0.2 \mathrm{mM}$ concentrations of YM598, only a few viable cells were seen in the two lines. There was no significant difference between the two lines (Fig. 3). These data suggest that YM598 may have a growth inhibition effect in the $0.01-0.05 \mathrm{mM}$ concentrations on both lines in vitro. In contrast, the cyto- 
Table 1. Top three up-regulated genes in highly metastatic cell lines in comparison with the parental low metastatic cell line

\begin{tabular}{|c|c|c|c|c|}
\hline Accession no. & Gene name & $\begin{array}{l}\text { Intensity in AZ-H5c, } \\
\text { AZ-P7a, or AZ-L5G }\end{array}$ & $\begin{array}{l}\text { Intensity } \\
\text { in AZ-521 }\end{array}$ & Ratio \\
\hline AZ-H5c vs. AZ521 & & AZ-H5c & & \\
\hline D11151 & Endothelin-A receptor & 987.6 & 27.3 & 30.1 \\
\hline L37127 & RNA polymerase II & 239.3 & 206.9 & 7.9 \\
\hline U43142 & Human vascular endothelial growth factor-related protein & 112.6 & -24.0 & 5.3 \\
\hline AZ-P7a vs. AZ521 & & AZ-P7a & & \\
\hline D11151 & Endothelin-A receptor & 1884 & 27.3 & 57.3 \\
\hline M35878 & Insulin-like growth factor-binding protein-3 & 739.8 & -25.4 & 20.4 \\
\hline S77154 & NGFI-B/nur77 $\beta$-type transcription factor homolog & 342.3 & 6.0 & 11.9 \\
\hline AZ-L5G vs. AZ521 & & AZ-L5G & & \\
\hline D11151 & Endothelin-A receptor & 2029 & 27.3 & 47.8 \\
\hline S77154 & NGFI-B/nur77 $\beta$-type transcription factor & 220.5 & 6.0 & 6.3 \\
\hline D50683 & TGF $\beta$-IIR $\alpha$ & 229.2 & 5.5 & 5.7 \\
\hline
\end{tabular}

AZ-521 AZ-H5c AZ-P7a AZ-L5G

ET-A

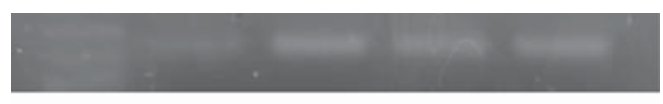

GAPDH

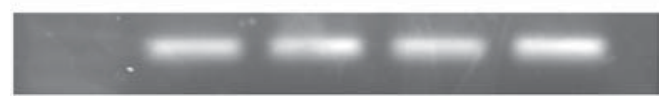

Fig. 2. Reverse transcription polymerase chain reaction (RTPCR) analysis of ET-A genes in the four cell lines. Electrophoretic images of ET-A mRNA expression by RT-PCR show more up-regulation of the ET-A gene in the highly metastatic cell lines than in the low metastatic AZ-521. Expression of the GAPDH gene was used as an internal control for the integrity of the RNA molecules

toxicity of YM598 was shown in the $0.1-0.2 \mathrm{mM}$ concentrations.

\section{Effect of YM598 on the tumor growth of AZ-H5c cells in vivo}

As shown in Fig. 4, the administration of YM598 inhibited growth of the AZ-H5c tumors significantly at any dosage. The mean tumor volumes at autopsy were $1453.9 \mathrm{~mm}^{3}$ (group 1), $502.7 \mathrm{~mm}^{3}$ (group 2), $448.0 \mathrm{~mm}^{3}$ (group 3), and $293.7 \mathrm{~mm}^{3}$ (group 4). The growth inhibition rates, compared with that of group 1, were $34.6 \%$ (group 2), 30.8\% (group 3), and 20.2\% (group 4). There was no evidence of gross toxicity, such as weight loss, resulting from YM598 treatment; and no death occurred during the experiment in any of the groups (Fig. 5).

\section{Effect of YM598 on the liver metastasis of AZ-H5c cells in vivo}

In the control group, five of six mice (metastatic rate $83.3 \%$ ) developed liver metastasis. On the other hand,

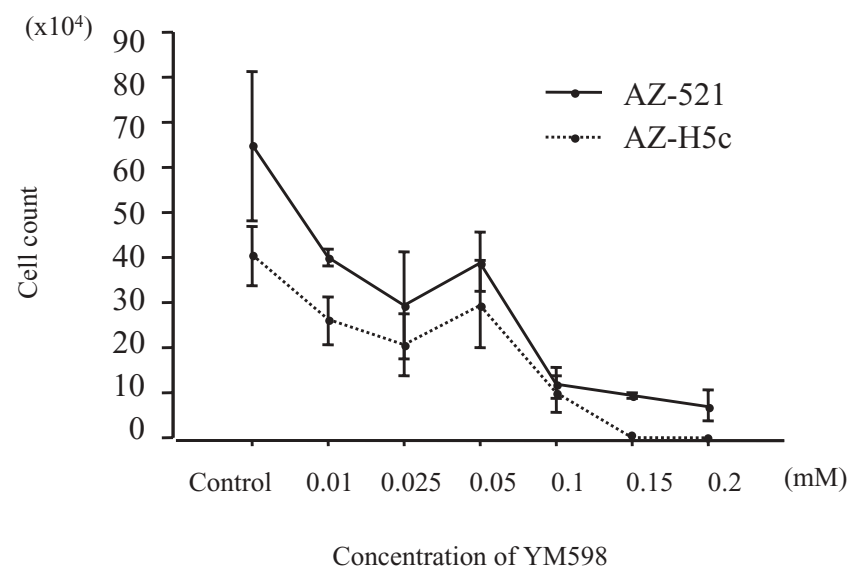

Fig. 3. Effect of YM598 on AZ-521 and AZ-H5c cell proliferation in vitro. After a 48-h incubation with YM598, the number of viable cells of both lines decreased at $0.01-0.05 \mathrm{mM}$ concentrations. At $0.1-0.2 \mathrm{mM}$ concentrations of YM598, only a few viable cells were seen in both lines. There was no significant difference between the two lines. The vertical bars show SEs

one of six mice in group $2(16.7 \%)$, one of five mice in group $3(20.0 \%)$, and one of six mice in group $4(16.7 \%)$ developed liver metastasis (Fig. 6). These results demonstrated that YM598 administration significantly reduced the liver metastatic rate of AZ-H5c. No advantage was obtained from dose escalation or pretreatment with YM598.

\section{Discussion}

With metastatic progression, gastric cancer is incurable, and existing systemic therapies are largely ineffective. Thus, the development of novel therapeutic strategies is needed. Differential gene expression analysis using a 


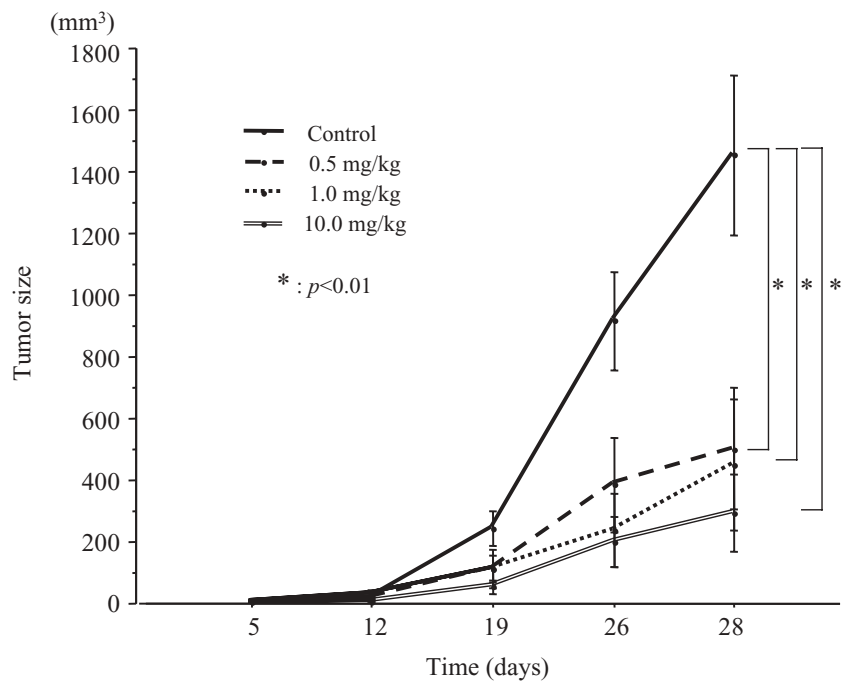

Fig. 4. Effect of YM598 on tumor growth of AZ-H5c in vivo. The administration of YM598 produced significant growth inhibition of the AZ-H5c tumors at any dosage. The vertical bars show SEs. $* P<0.01$ versus control

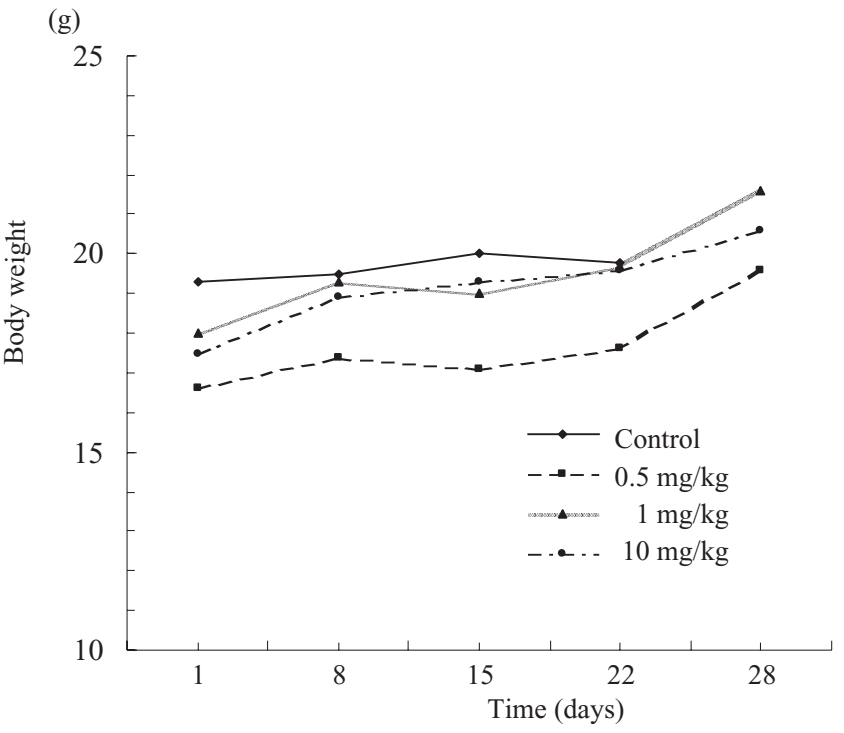

Fig. 5. Changes in the body weight of animals in an in vivo experiment of tumor growth. There was no evidence of weight loss resulting from YM598 treatment during the experiment in any group

DNA microarray is useful for selecting a candidate gene for cancer therapy. To select the metastasis-related genes of gastric cancer, we performed a microarray analysis of three highly metastatic gastric cancer cell lines and compared the results with those from a low metastatic parental cell line. The results suggested that the ET-A gene plays an important role in gastric cancer metastasis.

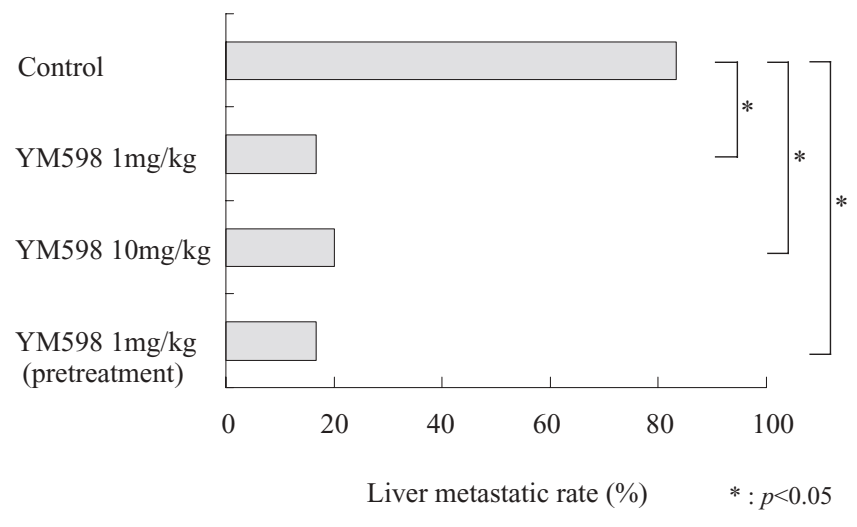

Fig. 6. Effect of YM598 on liver metastasis of AZ-H5c in vivo. YM598 significantly reduced the liver metastatic rate of AZ-H5c. No advantage was obtained from dose escalation or pretreatment

The family of ETs, including ET-1, ET-2, and ET-3, consists of 21 amino acids that mediate a variety of physiological functions. The effects of ETs on mammalian cells are mediated by two distinct subtypes of $G$ protein-coupled receptors: ET-A, which binds ET-1 and ET-2 with high affinity and ET-3 with low affinity; and ET-B, which binds all ET isopeptides with equal affinity [5]. Several human cancer cell lines produce ET-1, which has autocrine/paracrine growth factor functions [6,7]. Elevated plasma levels of ET-1 have also been detected in patients with various solid tumors, including hepatocellular [8] and colorectal [9] cancers. Furthermore, increased ET-A expression in malignant tissue has been demonstrated in several cancer types, including breast [10], ovarian [11], and advanced prostate [12] cancer. The engagement of ET-A by ET-1 is known to trigger activation of tumor growth [13], VEGF-induced angiogenesis [11], cancer cell invasiveness [14], and inhibition of apoptosis [15]. However, little is known regarding how the ET family is involved in cancer metastasis.

In this study, we investigated the effects of ET-A blockade on cell proliferation, tumor growth, and liver metastasis of a highly liver metastatic cell line, AZ-H5c. We used a non-peptide-selective ET-A antagonist, YM598, for the ET-A blockade. It is orally active and has high affinity and selectivity for ET-A with a long duration of action [4]. To date, the effects of the ET-A blockade on gastric cancer have not been investigated. The in vivo study demonstrated that YM598 had a significant growth inhibition effect on AZ-H5c at a dosage of $0.5-10.0 \mathrm{mg} / \mathrm{kg}$. YM598 also decreased the liver metastatic rate of AZ-H5c at $1 \mathrm{mg} / \mathrm{kg}$; however, no advantage was obtained from dose escalation or pretreatment. These results suggested that the liver metastasisinhibiting effect of YM598 could be largely achieved by 
growth inhibition of AZ-H5c. The mechanism through which ET-1 contributes to cell growth is thought to be as follows: Binding of ET-1 to ET-A in the plasma membrane triggers signal-transduction pathways through $\mathrm{Gq}$, a pertussis toxin-intensive $\mathrm{G}$ protein that is coupled to the ET-A intracellular domain. Activation of phospholipase $\mathrm{C}$, protein tyrosine kinases, and RAS ultimately results in activation of the RAF/MEK/MAPK pathway. Translocation of intracellular calcium $\left(\mathrm{Ca}^{2+}\right)$ and activation of protein kinase $\mathrm{C}$ and activated MAPK induce nuclear transcription of proto-oncogenes (e.g., c-FOS, c-JUN, and c- $M Y C$ ), leading to cell growth and mitogenesis [16]. Furthermore, ET-1 not only promotes cell growth, it suppresses apoptosis of cancer cells $[15,17]$. Therefore, ET-A antagonists could have the effects of both growth inhibition and apoptosis induction on carcinoma xenografts.

Tumor growth is one of multiple steps in a cancer metastatic pathway, and metastasis may possibly be inhibited by blocking one of these steps. In this study, YM598 markedly decreased liver metastasis, which might be due mainly to tumor growth inhibition. However, any other additional effect of the ET-A blockade (e.g., inhibition of cancer cell invasiveness or angiogenesis) could also contribute to reduction of the metastatic rate. Further studies are needed to define the definitive role of the ET-A system in gastric cancer metastasis. Considering its clinical usefulness, it should be determined if YM598 has additional antitumor effects in combination with chemotherapy or radiation therapy.

\section{Conclusion}

The present study demonstrated that ET-A blockade by YM598, a non-peptide-selective ET-A antagonist, is effective in inhibiting tumor growth and liver metastasis of a gastric cancer cell line in vivo. Further studies to assess the mechanisms of the effect and the clinical impact of ET-A blockade in gastric cancer are needed. These results suggest that ET-A blockade by YM598 could be a novel therapeutic strategy for inhibiting gastric cancer growth and metastasis.

Acknowledgments We thank Ms. Akiko Bizen (Department of Neurosurgery, Sapporo Medical University School of Medicine) for her skillful technical assistance, Mr. Katsuya Nakajima (GeneSpring Co., Ltd.) for his useful advice on statistical data analysis, Dr. Koji Yamaguchi and Dr. Hiroki Nomura (First Department of Surgery, Sapporo Medical University School of Medicine) for help with the animal studies, and Mr. Hironori Yuyama (Astellas Pharma, Inc.) for kindly providing the YM598.

\section{References}

1. Denno R, Yasoshima T, Hirata K, Kaya M, Fujinaga K, Ura H, et al. Tumorigenicity, motility and liver metastasis of human gastric carcinoma lines with high metastatic potential in liver of nude mice. Tumor Res 1995;30:57-65.

2. Nishimori H, Yasoshima T, Denno R, Shishido T, Hata F, Okada $\mathrm{Y}$, et al. A novel experiment mouse model of peritoneal dissemination of human gastric cancer cell: different mechanisms in peritoneal dissemination and hematogenous metastasis. Jpn J Cancer Res 2000;91:715-22.

3. Yamaguchi K, Ura H, Yasoshima T, Shishido T, Denno R, Hirata $\mathrm{K}$. Establishment and characterization of a human gastric carcinoma cell line that is highly metastatic to lymph nodes. J Exp Clin Cancer Res 2000;19:113-20.

4. Yuyama H, Sanagi M, Koakutsu A, Mori M, Fujimori A, Harada $\mathrm{H}$, et al. Pharmacological characterization of YM598, an orally active and highly potent selective endothelin $\mathrm{ET}_{\mathrm{A}}$ receptor antagonist. Eur J Pharmacol 2003;478:61-71.

5. Rubanyi GM, Polokoff MA. Endothelins: molecular biology biochemistry, pharmacology, physiology and pathophysiology. Pharmacol Rev 1994;46:325-415.

6. Kusuhara M, Yamaguchi K, Nagasaki K, Hayashi C, Suzuki A, Hori S, et al. Production of endothelin in human cancer cell lines. Cancer Res 1990;50:3257-61.

7. Shichiri M, Hirata Y, Nakajima T, Ando K, Imai T, Yanagisawa $\mathrm{M}$, et al. Endothelin-1 is an autocrine/paracrine growth factor for human cancer cell lines. J Clin Invest 1991;87:1867-71.

8. Nakamuta M, Ohashi M, Tabata S, Tanabe Y, Goto K, Naruse $\mathrm{M}$, et al. High plasma concentrations of endothelin-like immunoreactivities in patients with hepatocellular carcinoma. Am J Gastroenterol 1993;88:248-52.

9. Asham E, Shankar A, Loizidou M, Fredericks S, Miller K, Boulos $\mathrm{PB}$, et al. Increased endothelin-1 in colorectal cancer and reduction of tumour growth by $\mathrm{ET}_{\mathrm{A}}$ receptor antagonism. Br J Cancer 2001;85:1759-63.

10. Wulfing P, Gotte M, Sonntag B, Kersting C, Schmidt H, Wulfing $\mathrm{C}$, et al. Overexpression of endothelin-A-receptor in breast cancer: regulation by estradiol and cobalt-chloride induced hypoxia. Int J Oncol 2005;26:951-60.

11. Salani D, Di Castro V, Nicotra MR, Rosanò L, Tecce R, Venui A, et al. Role of endothelin-1 in neovascularization of ovarian carcinoma. Am J Pathol 2000;157:1537-47.

12. Gohji K, Kitazawa S, Tamada H, Katsuoka Y, Nakajima M. Expression of endothelin receptor a associated with prostate cancer progression. J Urol 2001;165:1033-6.

13. Bagnato A, Salani D, Di Castro V, Wu-Wong JR, Tecce R, Nicotra MR, et al. Expression of endothelin 1 and endothelin A receptor in ovarian carcinoma: evidence for an autocrine role in tumor growth. Cancer Res 1999;59:720-7.

14. Rosanò L, Varmi M, Salani D, Di Castro V, Spinella F, Natali PG, et al. Endothelin-1 induces tumor proteinase activation and invasiveness of ovarian carcinoma cells. Cancer Res 2001;61: 8340-6.

15. Wu-Wong JR, Chiou WJ, Wang J. Extracellular signal-regulated kinases are involved in the antiapoptotic effect of endothelin-1. J Pharmacol Exp Ther 2000;293:514-21.

16. Nelson JB, Bagnato A, Battistini B, Nisen P. The endothelin axis: emerging role in cancer. Nat Rev Cancer 2003;3:110-6.

17. Eberl LP, Valdenaire O, Saintgiorgio V, Jeannin JF, Jeanneret LJ. Endothelin receptor blockade potentiates FasL-induced apoptosis in rat colon carcinoma cells. Int J Cancer 2000;86: $182-7$. 